\title{
Expression of D0G1, CD117 \& PDGFRA in Gastrointestinal Stromal Tumours and Correlations with Clinicopathological Features \& Risk Assessment
}

\author{
Reshma Pallikkara Kunjunny', Muthukrishnan Chirayil Ponnappan² \\ 1, 2 Department of Pathology, Government Medical College, Kerala University of Health Sciences, \\ Thiruvananthapuram, Kerala, India.
}

\section{ABSTRACT}

\section{BACKGROUND}

Gastrointestinal stromal tumour (GIST) was first named in 1983. Gastrointestinal stromal tumours (GISTs) are a special kind of tumours which are derived from mesenchymal tissues of gastrointestinal tract and arises from the interstitial cells of Cajal, the pacemaker cells of the gastrointestinal (GI) tract responsible for the contractions of smooth muscles. ${ }^{1}$ Determination of the type of mutations in GIST plays a major role in assessing the risk of progression of the disease and also allows determination of the clinical management and treatment. More accurate GIST diagnosis is possible by using simultaneously various types of antibodies to immunohistochemistry methods in routine procedures. ${ }^{2}$

\section{METHODS}

In this descriptive cross sectional study expression of D0G1, CD117 \& PDGFRA was analysed in 70 patients with histopathologically diagnosed specimens of gastrointestinal stromal tumour, received in the Department of Pathology, Government Medical College, Thiruvananthapuram, using the immunohistochemical method.

\section{RESULTS}

On evaluating the CD117, DOG1 \& PDGFRA expression in GIST by immunohistochemistry showed $71.4 \%, 84.3 \%$ and $55.7 \%$ positivity respectively. Most of the patients fall in the age group of $60-70$ years with a slight male predominance. Most common location of GIST is stomach with tumour size of 5 $10 \mathrm{~cm}$. On microscopic evaluation spindle type GIST was predominant histopathological type. Considering the risk groups, histological type, mitotic count and tumour size, PDGFRA expression is more in low-risk groups. PDGFRA expression has insignificant relation with clinicopathologic features including age, sex, site of lesion, risk groups, histologic type, mitotic count and tumour size. Relationship between positive expression by CD117 \& DOG1 with risk group \& site of lesion are not statistically significant. When compared to the similar studies in literature, the obtained results are concordant.

\section{CONCLUSIONS}

Our study concluded that, $71.4 \%$ positive immunoreactions for CD117, 84.3\% positive immunoreactions for DOG1 \& $55.7 \%$ positive immunoreactions for PDGFRA. PDGFRA expression has insignificant relation with clinicopathologic features including age, sex, site of lesion, risk groups, histologic type, mitotic count and tumour size. Relationship between positive expression by CD117 \& DOG1 with risk group \& site of lesion are not statistically significant. The importance of this study is that PDGFRA expression in tumours can be considered for treatment by using tyrosine kinase inhibitors and avapritinib. So PDGFRA testing in GIST show a new path in the targeted therapy.

\section{KEY WORDS}

GIST (Gastrointestinal Stromal Tumour), Discovered on GIST 1(DOG1), Cluster of Differentiation (CD117), Platelet Derived Growth Factor Receptor A(PDGFRA), Anoctamin 1(ANO1), Succinate Dehydrogenase (SDH), Risk Group, Mitotic Count.
Corresponding Author: Dr. Muthukrishnan Chirayil Ponnappan, Associate Professor, Department of Pathology, Government Medical College, Thiruvananthapuram, Kerala, India. E-mail:drmkcp@yahoo.com

DOI: $10.14260 /$ jemds/2022/20

How to Cite This Article: Kunjunny RP, Ponnappan MC. Expression of DOG1, CD117 \& PDGFRA in gastrointestinal stromal tumours and correlations with clinicopathological features \& risk assessment. J Evolution Med Dent Sci 2022;11(01):103-108, 10.14260/jemds/2022/20

Submission 04-12-2021,

Peer Review 29-12-2021

Acceptance 05-01-2022,

Published 25-01-2022.

Copyright (c) 2022 Reshma Pallikkara Kunjunny et al. This is an open access article distributed under Creative Commons Attribution License [Attribution 4.0 International (CC BY 4.0)] 


\section{BACKGROUND}

Gastrointestinal stromal tumour (GIST), a kind of rare tumour, is the most common mesenchymal tumour in the gastrointestinal tract. ${ }^{3}$ The concept of stromal tumour was first proposed by Mazur \& Clark in 1983. Most GISTs have been previously classified as smooth muscles tumours (leiomyoma, leiomyosarcoma) or schwannoma due to incomplete understanding of its origin and differentiation. ${ }^{4-7}$ Presently, it is considered that GISTs originate from stem cells that differentiate toward interstitial cells of Cajal expressing tyrosine kinase receptors KIT or platelet derived growth factor receptor alpha (PDGFRA). ${ }^{8}$

The tumours can start anywhere in the GI tract, but they occur most often in the stomach $(60 \%)$ or the small intestine (35\%). Most of the rest are found in the oesophagus, colon \& rectum. A small number develop in the abdomen outside the GI tract. ${ }^{9-11}$

Localized GIST presents as a well circumscribed mass of highly variable size (ranging from incidental, submillimetre lesions to $>20 \mathrm{~cm}$ ). In larger lesions, the cut surface may show foci of haemorrhage, cystic change, and/or necrosis. Gastric GISTs often feature an intraluminal component \& may produce umbilicated mucosal ulcers. ${ }^{12}$ In the small bowel, GISTs present as external masses. Some GISTs feature a narrow pedicle linked to the serosal surface, the interruption of which may be contribute to the generation of extra gastrointestinal GISTs. Advanced disease most often presents as a main lesion associated with multiple smaller nodules that may extend from the diaphragm to the pelvis. Invasion of surrounding organs such as the spleen \& pancreas can be observed in aggressive tumours. SDH deficient GISTs are often associated with a distinctive multinodular pattern of growth. ${ }^{13-16}$

GIST is slightly more common in men. Although GISTs are most often diagnosed in people at 50 years of age or older, they can occur in any age group. ${ }^{17}$

Smaller GISTs are detected incidentally during endoscopy, surgery or CT. Advanced GISTs spread into the peritoneal cavity \& retroperitoneal space and often metastasize to the liver. Bone, skin \& soft tissue metastases are frequently observed, whereas lung metastases are exceedingly rare.18-19 Systemic spread can occur years after detection of the primary tumour. Gastric GISTs exhibit a higher local recurrence rate than do small bowel GISTs, but the latter have a higher rate of abdominal dissemination \& metastasis. Incidental subcentimetre GISTs (Called microGIST) seem to be remarkably common. Approximately $25 \%$ of gastric GISTs (excluding microGISTs) are clinically malignant.20-21

The two most specific \& sensitive diagnostic biomarkers for GIST are protein expression of KIT (CD117 - a transmembrane tyrosine kinase receptor) and anoctamin 1 (ANO 1) (DOG 1), which are positive in about 95\% of all GIST. ${ }^{22}$

DOG1 is a calcium dependent chloride channel protein. It has many significant functions such as regulation of the cholinergic activity of gastrointestinal smooth muscle \& regulation of both the survival \& proliferation of cells. Its expression indicates tumourigenesis. ${ }^{23}$

A small subset of GIST stain negative for both KIT \& ANO1. The combined detection of GISTs CD117 \& PDGFRA can provide accurate diagnose evidences both in pathology \& in clinic. ${ }^{24}$ The probability that DOG1 may be a potential target for GISTs therapy makes it possible to develop new targeted drugs and to identify the patients who can benefit from this therapy. ${ }^{25}$

Most syndromic GISTs are SDH - deficient, including those associated with the non-hereditary Carney triad (GIST, pulmonary chondroma, paraganglioma) and the autosomal dominant Carney-Stratakis syndrome (GIST \& paraganglioma in the context of SDH germline mutations). Rarely, GISTs are associated with neurofibromatosis type 1 (NF1); such cases are often multifocal \& most are located in the small bowel. The extremely rare familial GISTs are caused by germline mutation of KIT or PDGFRA. ${ }^{26-29}$

\section{Objectives}

To find out the proportions of GIST specimen with expression of DOG1, CD117 \& PDGFRA was our primary objective. Secondary objective was to find out correlations with clinicopathological features \& risk assessment.

\section{METHODS}

Descriptive cross sectional study was conducted at the Department of Pathology, Government Medical College, Thiruvananthapuram. Study period was one year from date of approval. A 5-year analysis of histopathologically proved gastrointestinal stromal tumours received in a period of 20162019 was done.

\section{Sample Technique and Size}

Consecutive

Sample size is calculated using the formula-

$n=(1.96) 2 q / d 2$

$\mathrm{p}$ is prevalence of expression of PDGFRA in gastrointestinal stromal tumour, from previous studies. ${ }^{1}$

$\mathrm{q}$ is $100-\mathrm{p} \mathrm{p}=58.2 \mathrm{q}=41.8$

$\mathrm{d}$ is $20 \%$ of $\mathrm{p} \mathrm{d}=20 \times 58.2 / 100=11.64$

$\mathrm{n}=1.96 \times 1.96 \times 58.2 \times 41.8 / 11.64 \times 11.64$

$=68.97$

70 specimens will be included in the study

\section{Study Variables}

1. Expression of DOG1, CD117 \& PDGFRA.

2. Age, sex, site of lesion, tumour size, mitotic count, histology type, and risk group.

\section{Data Collection Tool}

1. Formalin fixed paraffin embedded tissue blocks

2. Haematoxylin and eosin-stained sections.

3. IHC marker- DOG1, CD117, PDGFRA

4. Hospital records.

5. A structural proforma will be used to collect data

\section{Data Collection Technique}

All the specimens are fixed in 10\% neutral buffered formalin. After the process of cutting, bitting, tissue processing, paraffin embedded blocks are made. Sections of 4-5 micrometre 
thickness are cut from the blocks and stained with haematoxylin eosin staining. The slides are analysed under optical microscope and diagnosis is made as per WHO classification. Immunohistochemistry performed on all diagnosed specimens of GIST with DOG1, CD117 and PDGFRA. Risk score will be done based on tumour size and mitotic index.

\section{Statistical Analysis}

Data will be entered into excel sheet and analysis to be done using SPSS software version. Qualitative variables such as sex, site of lesion, risk group, histology type, CD117, DOG1 \& PDGFRA expression will be expressed as proportion and quantitative variables such as age, tumour size, and mitotic index will be expressed as mean and standard deviation. Analysis of data will be done using chi-square test.

\section{RESULTS}

The expression of DOG1, CD117 \& PDGFRA in GIST was studied and also evaluated whether there is any significant relation between PDGFRA positivity and clinicopathological characters like age, sex, site of lesion, risk groups, tumour size, histological type and mitotic count. Also find out any correlation with CD117 \& DOG1 positivity with risk group and site of tumour.

On evaluating the CD117, D0G1 \& PDGFRA expression in GIST by immunohistochemistry showed $71.4 \%$ (Refer Table 1 \& Figure 1, 2, 3), 84.3\% (Refer Table 2 \& Figure 4, 5) and 55.7\% (Refer Table 3 \& Figure 6) positivity respectively.
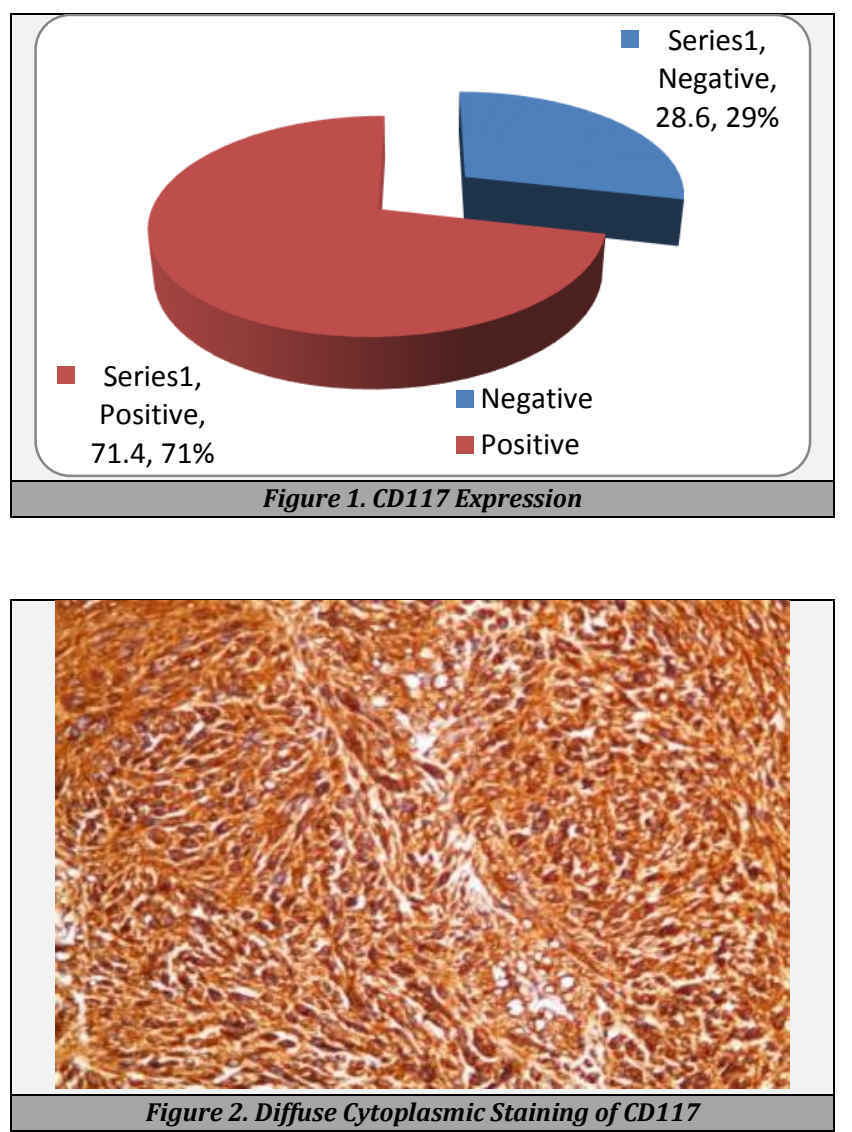

\begin{tabular}{|ccc|}
\hline CD117 & Count & Percent \\
Negative & 20 & 28.6 \\
Positive & 50 & 71.4 \\
\hline \multicolumn{3}{|c}{ Table 1. CD117 Expression } \\
\hline
\end{tabular}

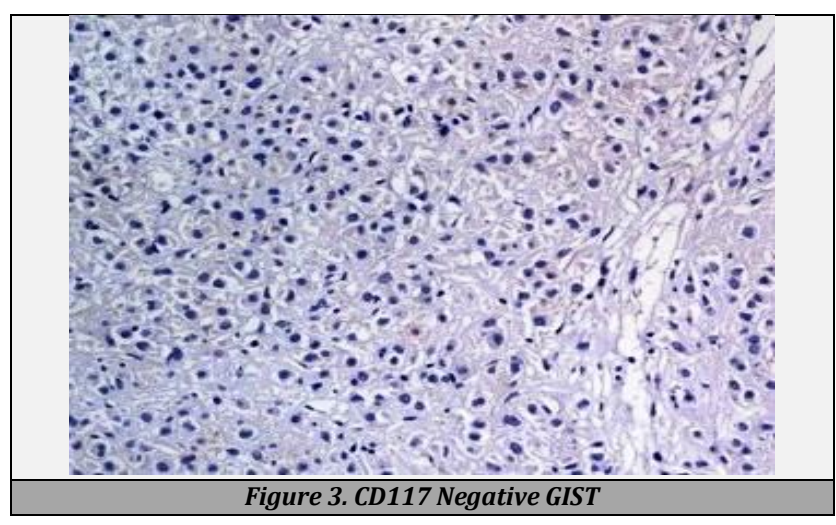

\begin{tabular}{|ccc|}
\hline DOG1 & Count & Percent \\
Negative & 11 & 15.7 \\
Positive & 59 & 84.3 \\
\hline & Table 2. DOG1 Expression \\
\hline
\end{tabular}
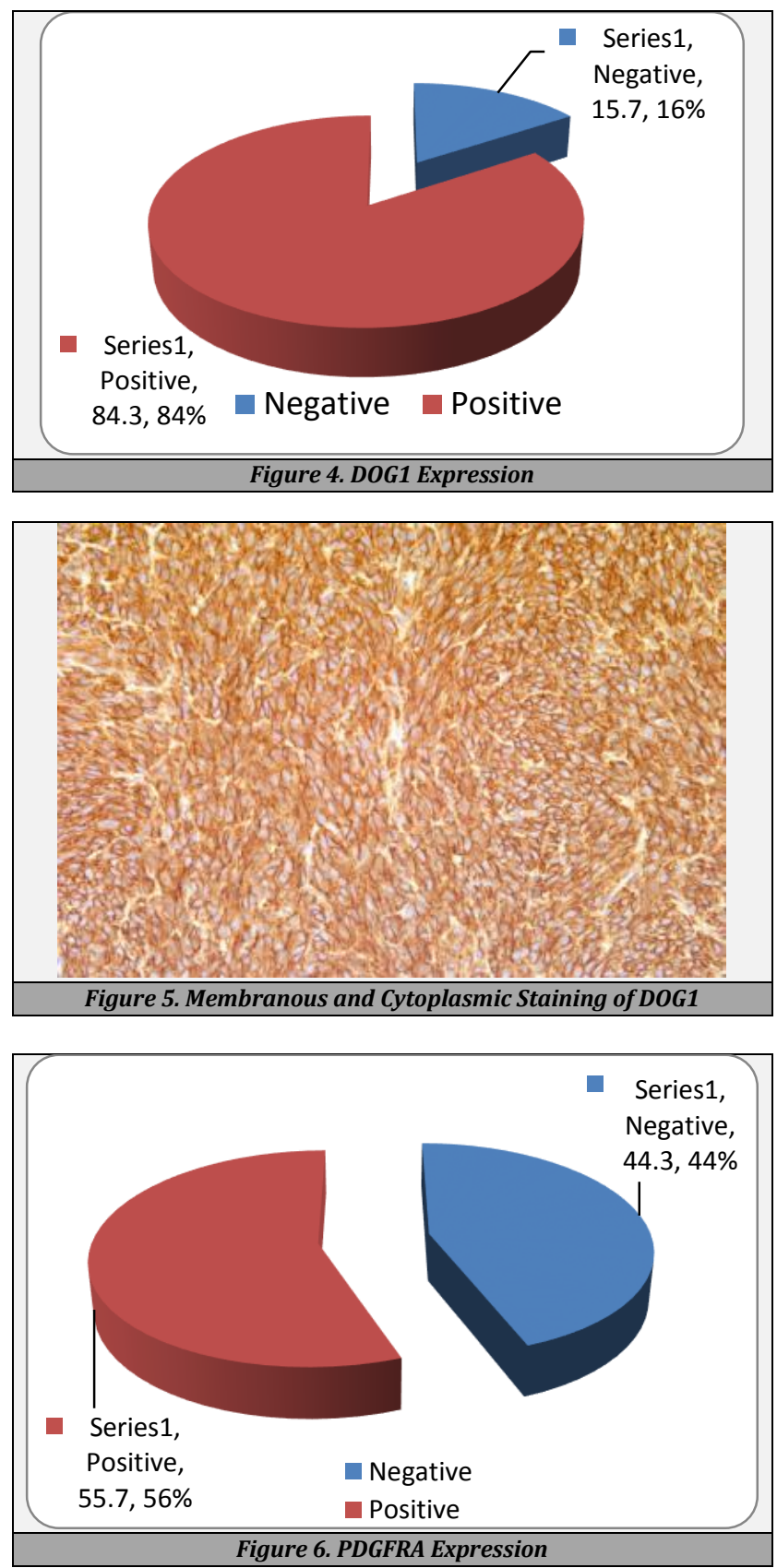
Most of the patients fall in the age group of $60-70$ years with a slight male predominance. Most common location of GIST is stomach (Refer Table 4 \& Figure 7,8 ) with tumour size of $5-10 \mathrm{~cm}$.

\begin{tabular}{|c|c|c|}
\hline PDGFRA & Count & Percent \\
\hline Negative & 31 & 44.3 \\
\hline Positive & 39 & 55.7 \\
\hline \multicolumn{3}{|c|}{ Table 3. PDGFRA Expression } \\
\hline Site of Tumour & Count & Percent \\
\hline Stomach & 47 & 67.1 \\
\hline Small intestine & 18 & 25.7 \\
\hline Large intestine & 1 & 1.4 \\
\hline Pancreas & 1 & 1.4 \\
\hline Mesentery & 3 & 4.3 \\
\hline
\end{tabular}
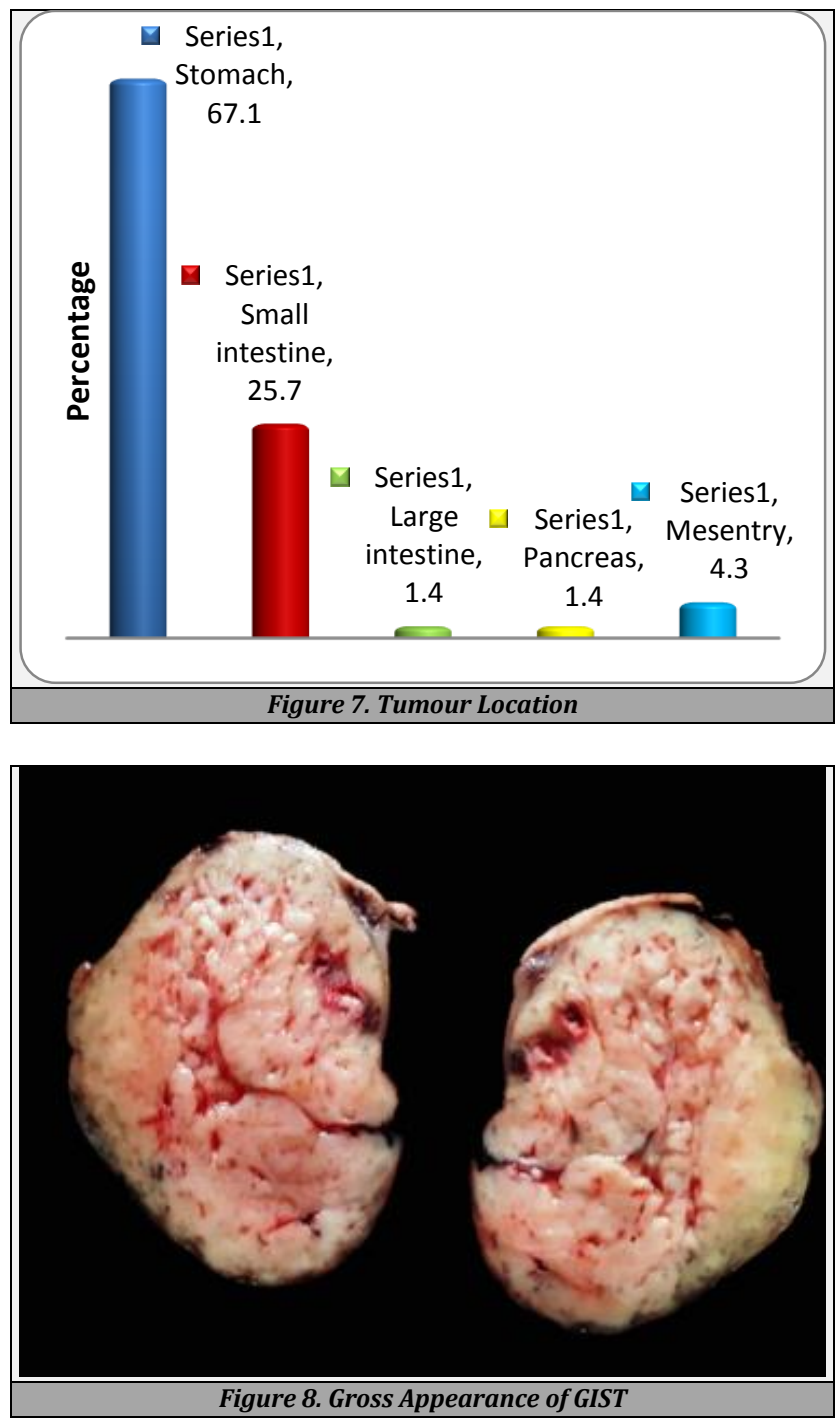

On microscopic evaluation spindle type GIST was predominant histopathological type (Refer Table 5 \& Figure 9, 10).

\begin{tabular}{|ccc|}
\hline Histological Type & Count & Percent \\
Spindle & 61 & 87.1 \\
Epithelioid & 6 & 8.6 \\
Mixed & 3 \\
\hline \multicolumn{3}{|c|}{ Table 5. Histologic Type } \\
\hline
\end{tabular}
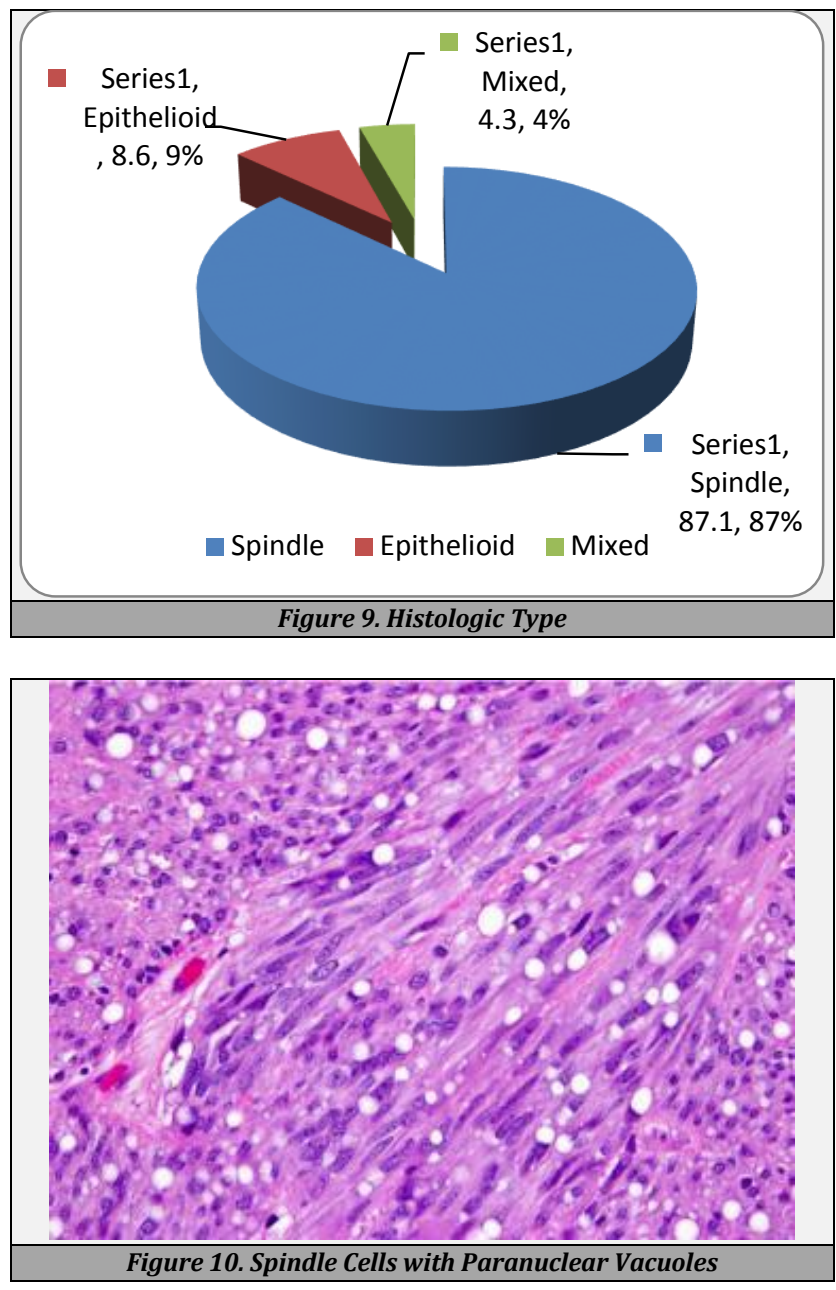

Majority of GIST cases comes under intermediate risk groups (35.7\%), followed by low risk (30\%) and high risk (28.6\%) groups respectively.

Considering the risk groups, histological type, mitotic count and tumour size, PDGFRA expression is more in low-risk groups (68\%), epithelioid and mixed type (66.7\%) of GIST, 5 10 mitotic counts per $50 \mathrm{HPF}(66.7 \%)$ and $>10 \mathrm{~cm}$ tumour size $(63.2 \%)$ respectively. PDGFRA expression has insignificant relation with clinicopathologic features including age, sex, site of lesion, risk groups, histologic type, mitotic count and tumour size (Refer Table 7). Relationship between positive expression by CD117 \& DOG1 with risk group \& site of lesion are not statistically significant as the p values are 0.211 and $0.317(>0.05)$ respectively. (Refer Table 6 ). When compared to the similar studies in literature, the obtained results are concordant.

\begin{tabular}{|c|c|c|c|c|c|c|c|}
\hline & & \multicolumn{2}{|c|}{$\begin{array}{l}\text { Either or both } \\
\text { CD117 \& DoG1 } \\
\text { Negative }\end{array}$} & \multicolumn{2}{|c|}{$\begin{array}{l}\text { Both CD117 \& } \\
\text { DoG1 Positive }\end{array}$} & \multirow[t]{2}{*}{$\chi^{2}$} & \multirow[t]{2}{*}{$\mathbf{P}$} \\
\hline & & Count & Percent & Count & Percent & & \\
\hline \multirow{3}{*}{$\begin{array}{l}\text { Risk } \\
\text { groups }\end{array}$} & Very low/ low risk & 12 & 48.0 & 13 & 52.0 & \multirow{3}{*}{3.11} & \multirow{3}{*}{0.211} \\
\hline & Intermediate risk & 12 & 48.0 & 13 & 52.0 & & \\
\hline & High risk & 5 & 25.0 & 15 & 75.0 & & \\
\hline \multirow{3}{*}{$\begin{array}{l}\text { Site of } \\
\text { lesion }\end{array}$} & Stomach & 21 & 44.7 & 26 & 55.3 & \multirow{3}{*}{2.3} & \multirow{3}{*}{0.317} \\
\hline & Small intestine & 5 & 27.8 & 13 & 72.2 & & \\
\hline & Others & 3 & 60.0 & 2 & 40 & & \\
\hline
\end{tabular}




\begin{tabular}{|c|c|c|c|c|c|c|}
\hline & Variable & Number & rPositive & ePercentage & $\chi^{2}$ & $\mathbf{P}$ \\
\hline \multirow{3}{*}{ Age } & $<=40$ & 10 & 6 & $60.0 \%$ & \multirow{3}{*}{2.6} & \multirow{3}{*}{0.272} \\
\hline & $41-60$ & 29 & 19 & $65.5 \%$ & & \\
\hline & $>60$ & 31 & 14 & $45.2 \%$ & & \\
\hline \multirow{2}{*}{ Sex } & Male & 38 & 23 & $60.5 \%$ & \multirow{2}{*}{0.78} & \multirow{2}{*}{0.377} \\
\hline & Female & 32 & 16 & $50.0 \%$ & & \\
\hline \multirow{3}{*}{ Site of lesion } & Stomach & 47 & 27 & $57.4 \%$ & \multirow{3}{*}{0.56} & \multirow{3}{*}{0.757} \\
\hline & Small intestine & 18 & 10 & $55.6 \%$ & & \\
\hline & Others & 5 & 2 & $40.0 \%$ & & \\
\hline \multirow{3}{*}{ Risk groups } & Very low/ low risk & 25 & 17 & $68.0 \%$ & \multirow{3}{*}{2.6} & \multirow{3}{*}{0.273} \\
\hline & Intermediate risk & 25 & 13 & $52.0 \%$ & & \\
\hline & High risk & 20 & 9 & $45.0 \%$ & & \\
\hline \multirow{3}{*}{ Histologic type } & Spindle & 61 & 33 & $54.1 \%$ & \multirow{3}{*}{0.5} & \multirow{3}{*}{0.778} \\
\hline & Epithelioid & 6 & 4 & $66.7 \%$ & & \\
\hline & Mixed & 3 & 2 & $66.7 \%$ & & \\
\hline \multirow{3}{*}{ Mitotic count } & $<5$ & 47 & 26 & $55.3 \%$ & \multirow{3}{*}{1.06} & \multirow{3}{*}{0.590} \\
\hline & $5-10$ & 12 & 8 & $66.7 \%$ & & \\
\hline & $>10$ & 11 & 5 & $45.5 \%$ & & \\
\hline \multirow{4}{*}{ Tumour size } & $<2$ & 2 & 1 & $50.0 \%$ & \multirow{4}{*}{0.59} & \multirow{4}{*}{0.744} \\
\hline & $2-5$ & 19 & 10 & $52.6 \%$ & & \\
\hline & $5-10$ & 30 & 16 & $53.3 \%$ & & \\
\hline & $>10$ & 19 & 12 & $63.2 \%$ & & \\
\hline Table 7. & $\begin{array}{r}\text { Relationship } \\
\text { Clinicopa }\end{array}$ & $\begin{array}{l}\text { between } \\
\text { thologic }\end{array}$ & $\begin{array}{l}\text { PDGFRA } \\
\text { Features }\end{array}$ & $\begin{array}{l}\text { A Expressi } \\
S\end{array}$ & and & \\
\hline
\end{tabular}

\section{DISCUSSION}

GISTs are common kind of tumours derived from gastrointestinal mesenchyme, which is presented as spindle and epithelial cells, and sometimes pleomorphic cells arranged in cords or diffuse histologically. GISTs express the gene product KIT (CD117) of c-Kit, and are a mutant c-Kit or PDGFRA induced tumour. CD117 can be a target for therapy, and the use of TKIs for the inhibition of KIT and PDGFRA kinase has revolutionized the treatment of GISTs, so this specific diagnose is a potential therapy of patients. Various prognostic factors for determining the survival for GIST include location, tumour size, risk group, mitotic rate and mutational status.

The present study included 70 GIST specimens received in the Department of Pathology, Government Medical College, Thiruvananthapuram from 2016 to 2019.

Out of 70 cases taken, most of the cases are in the age group of $60-70$ years and shows a slight male predominance.

The most common location of tumour in the present study is the stomach. All other studies showed a similar finding except one study by Beril guler et al. in that small intestine is the most common site.

In the present study most common histological type of GIST is spindle type. All other studies showed similar findings.

Tumour size and mitotic rate have been accepted as the most reliable parameters by many authors for determining prognosis. The number of mitosis indicates active growth in the tumour. Tumour size and mitotic rate are used for categorizing the risk groups. 5 or fewer mitosis/50 HPF is associated with low- risk groups. In the present study majority of GIST cases have $5-10 \mathrm{~cm}$ in size, mitotic count $<5 / 50 \mathrm{hpf}$ and belongs to intermediate risk category. All other studies showed a similar finding except one study by Beril guler et al. in that majority cases belongs to high-risk category (54.1\%).

The study of expression of DOG1, CD117 \& PDGFRA in GIST was the primary objective of the present study.

The CD117, D0G1 \& PDGFRA positivity in various studies ranges between 70 to $95 \%$ (CD117), 85 to $90 \%$ (DOG1) and 53 to $80 \%$ (PDGFRA) respectively. All studies showed increased positivity of CD117 \& DOG1. The present study also shows the similar finding.
The secondary objective of the present study was to evaluate correlation between PDGFRA positivity and clinicopathological characters like age, sex, site of lesion, risk groups, tumour size, histological type and mitotic count. Also find out any correlation with CD117 \& DOG1 positivity with risk group and site of tumour. The evaluation is done by calculating $\mathrm{p}$ value through chi square test. Relationship between positive expression by CD117 \& DOG1 with risk group \& site of lesion are not statistically significant as the $p$ values are 0.211 and $0.317(>0.05)$ respectively.

PDGFRA expression has insignificant relation with clinicopathologic features including age, sex, site of lesion, risk groups, histologic type, mitotic count and tumour size. The $p$ value for age, sex, site of lesion, risk groups, histologic type, mitotic count and tumour size in the present study is in concordance with previous studies.

\section{The Observations Made in the Present Study Are}

1. The common age prevalence of GISTs falls under $60-70$ years.

2. There is a slight male predominance.

3. Most common location of tumour is noted in stomach.

4. The most common histological type is spindle type.

5. Most of GISTs are comes under intermediate risk group.

6. Majority of tumours shows $5-10 \mathrm{~cm}$ in size and mitotic count $<5 / 50$ HPF.

7. The important observation made is that of expression of CD117, DOG1 and PDGFA, showed $71.4 \%, 84.3 \%$ and $55.7 \%$ respectively.

8. Considering the risk groups, histological type, mitotic count and tumour size, PDGFRA expression is more in low-risk groups.

9. There is no significant relation with clinicopathologic features including age, sex, site of lesion, risk groups, histologic type, mitotic count and tumour size.

\section{CONCLUSIONS}

Our study concluded that, $71.4 \%$ positive immunoreactions for CD117, 84.3\% positive immunoreactions for DOG1 \& $55.7 \%$ positive immunoreactions for PDGFRA. PDGFRA expression has insignificant relation with clinicopathologic features including age, sex, site of lesion, risk groups, histologic type, mitotic count and tumour size. Relationship between positive expression by CD117 \& DOG1 with risk group \& site of lesion are not statistically significant. The importance of this study is that PDGFRA expression in tumours can be considered for treatment by using tyrosine kinase inhibitors and avapritinib. So PDGFRA testing in GIST shows a new path in the targeted therapy.

Data sharing statement provided by the authors is available with the full text of this article at jemds.com.

Financial or other competing interests: None.

Disclosure forms provided by the authors are available with the full text of this article at jemds.com.

\section{REFERENCES}

[1] Miettinen M, Lasota J. Gastrointestinal stromal tumours, review on morphology, molecular pathology, prognosis, 
and differential diagnosis. Arch Pathol Lab Med 2006;130(10):1466-78.

[2] Sun XW, Feng ZJ, Huang P, et al. Expression of DOG1, CD117 and PDGFRA in gastrointestinal stromal tumours and correlations with clinicopathology. Asian Pacific Journal of Cancer Prevention 2012;13:1389-93.

[3] Ríos-Morenoa MJ, Jaramilloa S, Gallardoa SP, et al. Gastrointestinal stromal tumours (GISTs): CD117, DOG-1 and PKC $\theta$ expression. Is there any advantage in using several markers? Pathol Res Pract 2012;208(2):74-81.

[4] Lasota J, Miettinen M. Clinical significance of oncogenic KIT and PDGFRA mutations in gastrointestinal stromal tumours. Histopathology 2008;53(3):245-66.

[5] Xu C, Han H, Wang J, et al. Diagnosis value of CD117 and PDGFRA, alone or in combination DOG1, as biomarkers for gastrointestinal stromal tumours. Ann Transl Med 2015;3(20):308.

[6] Rizzardi C, Marzinotto S, Avellini C, et al. A KIT-negative, DOG1-positive epithelioid GIST of the stomach harboring a novel PDGFRA exon 14 single nucleotide deletion. Anticancer Res 2012;32(5):1775-8.

[7] Miettinen M, Sobin LH, Sarlomo-Rikala M. Immunohistochemical spectrum of GISTs at different sites and their differential diagnosis with a reference to CD117 (KIT). Mod Pathol 2000;13(10):1134-42.

[8] Swalchick W, Shamekh R, Bui MM. Is DOG1 immunoreactivity specific to gastrointestinal stromal tumour? Cancer Control 2015;22(4):498-504.

[9] Arne G. Expression profiling of gastrointestinal stromal tumours biomarkers for prognosis and therapy. Sahlgrenska Cancer Center, Department of Pathology, Sahlgrenska Academy at the University of Gothenburg, Gothenburg, Sweden 2012.

[10] Reichardt P, Morosi C, Wardelmann E, et al Gastrointestinal stromal tumours: evolving role of the multidisciplinary team approach in management. Expert Rev Anticancer Ther 2012;12(8):1053-68.

[11] Rubin BP, Heinrich MC, Corless CL Gastrointestinal stromal tumour. Lancet 2007;369:1731-41.

[12] Rubin BP, Singer S, Tsao C, et al KIT activation is a ubiquitous feature of gastrointestinal stromal tumours. Cancer Res 2001;61(22):8118-21.

[13] Blay JY, Le Cesne A, Cassier PA, et al. Gastrointestinal stromal tumours (GIST): a rare entity, a tumour model for personalized therapy, and yet ten different molecular subtypes. 2012;13(72):357-67.

[14] Miettinen M, Lasota J, Sobin LH. Gastrointestinal stromal tumours of the stomach in children and young adults: a clinicopathologic, immunohistochemical, and molecular genetic study of 44 cases with long-term follow-up and review of the literature. Am J Surg Pathol 2005;29(10):1373-81.
[15] Hoda KM, Rodriguez SA, Faigel DO. EUS-guided sampling of suspected GI stromal tumours. Gastrointest Endosc 2009;69(7):1218-23.

[16] Miettinen M, Wang ZF, Lasota J. DOG1 antibody in the differential diagnosis of gastrointestinal stromal tumours: a study of 1840 cases. Am J Surg Pathol 2009;33(9):1401-8.

[17] Hwang DG, Qian X, Hornick JL. DOG1 antibody is a highly sensitive and specific marker for gastrointestinal stromal tumours in cytology cell blocks. Am J Clin Pathol 2011;135(3):448-53.

[18] Fletcher CD, Berman JJ, Corless C, et al. Diagnosis of gastrointestinal stromal tumours: a consensus approach. Hum Pathol 2002;33(5):459-65.

[19] Faigel DO, Abulhawa S. Gastrointestinal stromal tumours: the role of the gastroenterologist in diagnosis and risk stratification. J Clin Gastroenterol 2012;46(8):629-36.

[20] Liu X, Ma D, Wu L, et al. Expression and clinical significance of c-kit oncogene in gastrointestinal stromal tumours. Zhonghua Wai Ke Za Zhi 2002;40(4):277-9.

[21] Qinling S, Wang H, Xiuwei S. DOG1, CD117 and PDGFRA expression and correlation in gastrointestinal stromal tumour. World Chinese Journal of Digestology 2011;19(9):912-8.

[22] Heinrich MC, Corless CL, Duensing A, et al. PDGFRA activating mutations in gastrointestinal stromal tumours. Science 2003;299(5607):708-10.

[23] Novelli M, Rossi S, Rodriguez-Justo M, et al. DOG1 and CD117 are the antibodies of choice in the diagnosis of gastrointestinal stromal tumours. Histopathology 2010;57(2):259-70.

[24] Liegl B, Hornick JL, Corless CL, et al. Monoclonal antibody DOG1.1 shows higher sensitivity than KIT in the diagnosis of gastrointestinal stromal tumours, including unusual subtypes. Am J Surg Pathol 2009;33(3):437-46.

[25] Dei Tos AP. The reappraisal of gastrointestinal stromal tumours: from Stout to the KIT revolution. Virchows Arch 2003;442(5):421-8.

[26] Ma PB, Neveu CR. Gastrointestinal stromal tumours. An update. Rev Med Chil 2008;136(7):921-9.

[27] Corless CL, Heinrich MC. Molecular pathobiology of gastrointestinal stromal sarcomas. Annu Rev Pathol 2008;3:557-86.

[28] Dow N, Giblen G, Sobin LH, et al. Gastrointestinal stromal tumours: differential diagnosis. Semin Diagn Pathol 2006;23(2):111-9.

[29] Espinosa I, Lee $\mathrm{CH}$, Kim MK, et al. A novel monoclonal antibody against DOG1 is a sensitive and specific marker for gastrointestinal stromal tumours. Am J Surg Pathol 2008;32(2):210-8. 